\title{
Establishing a protocol for the selection of zircon inclusions in garnet for Raman thermobarometry :
}

\section{Nicola Campomenosi ${ }^{1, *}$, Daniela Rubatto ${ }^{2}$, Joerg Hermann ${ }^{2}$, Boriana Mihailova ${ }^{3}$, Marco Scambelluri ${ }^{1}$, and Matteo Alvaro ${ }^{4}$}

\author{
${ }^{1}$ Department of Earth Science, Environment \& Life, University of Genoa, Corso Europa 26, 16132 Genoa, Italy \\ ${ }^{2}$ Institute of Geological Sciences, University of Bern, Baltzerstrasse 1+3 3012 Bern, Switzerland \\ ${ }^{3}$ Department of Earth Sciences, University of Hamburg, Grindelallee 48, D-20146 Hamburg, Germany \\ ${ }^{4}$ Department of Earth and Environmental Sciences, University of Pavia, Via A. Ferrata, 127100 Pavia, Italy
}

\begin{abstract}
The structural and chemical properties of zircon inclusions in garnet megablasts from the Dora Maira Massif (Western Alps, Italy) were characterized in detail using charge contrast imaging, Raman spectroscopy, and laser ablation inductively coupled plasma mass spectrometry (LA-ICP-MS). The aim of this work is to determine to what extent the degree of metamictization, metamorphic recrystallization, inherent structural heterogeneity, chemical composition, and zoning, along with the elastic stress imposed by the host mineral, can influence the Raman peak position of the zircon inclusion and hence, the residual pressure estimated via Raman geo-thermobarometry. We show and confirm that metamictization and inherent structural heterogeneity have a major influence in the Raman spectra of zircon in terms of peak position and peak width. We suggest that, for spectral resolution of $2 \mathrm{~cm}^{-1}$, the peak width of the $B_{1 \mathrm{~g}}$ mode near $1008 \mathrm{~cm}^{-1}$ of reliable grains must be smaller than $5 \mathrm{~cm}^{-1}$. The method can be applied to both inherited igneous and newly formed Alpine metamorphic crystals. By coupling structural and chemical information, we demonstrate that there are no significant differences between the Raman spectra of zircon with oscillatory-zoned texture, formed during magmatic crystallization, and those formed by fluid-induced Alpine (re)crystallization. The discrimination between magmatic and metamorphic zircon based only on micro-textural constraints is not robust. Finally, our results allow establishing a protocol devoted to the selection of reliable buried zircon inclusions, relying only on Raman spectroscopic measurements, to use for elastic thermobarometry applications.
\end{abstract}

Keywords: Dora Maira Massif, elastic thermobarometry, zircon, metamictization

\section{INTRODUCTION}

Zircon is one of the most common accessory minerals in various igneous, sedimentary, and metamorphic rocks. Furthermore, due to its large stability field and its physical robustness, zircon often hosts ultrahigh pressure (UHP) metamorphic minerals such as coesite and diamond (Parkinson and Katayama 1999; Ye et al. 2000b; Hermann et al. 2001; Rubatto and Hermann 2007; Katayama and Maruyama 2009). Zircon can be used for a wide range of applications, including U-Pb geochronology, and potential use as a host phase for the disposal of excess weapons-grade $\mathrm{Pu}$ because of its capacity to incorporate radioactive elements, (e.g., Ewing et al. 1995). Further, radiation-damaged zircon has been successfully applied as a model system to elucidate the behavior of partially ordered structures at high pressure and temperature conditions (e.g., Colombo et al. 1999; Binvignat et al. 2018).

A recent development in zircon petrology is its exploitation as a mineral inclusion in the frame of Raman elastic thermobarometry (Campomenosi et al. 2018; Stangarone et al. 2019; Zhong et al. 2019). The main idea is that the geochemical and isotopic information that can be obtained from zircon is complemented by elastic thermobarometry of zircon and its host to retrieve $P$-T-time-fluid-deformation paths of metamorphic and igneous

* E-mail: nicola.campomenosi@edu.unige.it

Open access: Article available to all readers online. rocks. For this new application, however, zircon composition and structural metamictization due to the decay of radioactive elements such as $U$ and Th within its crystal structure has to be taken into account, because the radiation-induced structural changes can lead to large variations in the Raman spectrum. Indeed, the Raman peaks of radiation-damaged crystals shift toward lower wavenumbers, while the peak width increases with respect to pristine crystals taken as reference (Colombo et al. 1999; Ríos et al. 2000; Nasdala et al. 2001; Geisler and Pidgeon 2002; Binvignat et al. 2018). In this regard, useful diagrams such as peak width $(\Gamma)$, in terms of full-width at half maximum, vs. peak position of the major Raman peak near $1008 \mathrm{~cm}^{-1}\left(\mathrm{~B}_{\mathrm{lg}}\right.$ phonon mode related to antisymmetric $\mathrm{SiO}_{4}$ stretching) can be exploited to recognize the zircon crystals whose Raman spectra is unaffected by metamictization processes and that can be safely used for elastic thermobarometry purposes (e.g., Zhong et al. 2019). However, even though the degree of metamictization is the main factor affecting the Raman spectra of zircon, it is not the only one. Cathodoluminescence (CL) studies of zircon internal zoning (e.g., Rubatto and Gebauer 2000; Corfu et al. 2003) reveal that single crystals of zircon, particularly in metamorphic rocks, commonly show a complex internal chemical and structural heterogeneity at the micrometer-scale. Furthermore, variations in the Raman peak positions can be caused also by chemical substitution: a typical example is $\mathrm{Hf}$ replacing $\mathrm{Zr}$ at the dodeca- 
hedral crystallographic site (e.g., Hoskin and Rodgers 1996).

To address these problems, we have chosen a particularly well-known sample suite from the Dora Maira UHP unit (Western Alps, Italy), where garnet megablasts host abundant zircon inclusions. We report micrometer-scale structural and chemical information of partially exposed zircon crystals studied by complementary Raman spectroscopy, charge contrast (CC) imaging, and laser ablation inductively coupled plasma mass spectrometry (LA-ICP-MS). The purpose is (1) to determine quantitatively how the structural and chemical heterogeneities of zircons can affect their Raman spectrum and (2) to propose a best-practice protocol for the selection of zircon inclusions suitable for elastic thermobarometric estimations.

\section{SAMPLE DESCRIPTION}

We selected exposed and buried zircon inclusions in garnet megablasts from the UHP whiteschists of the Dora Maira Massif. The investigated samples come from the two major outcrops of the Brossasco-Isasca Unit (e.g., Chopin 1984): Vallone Gilba and Vallone Martiniana close to the Case Fapina and Case Parigi localities, respectively. Several polished sections of about $350 \mu \mathrm{m}$ in thickness have been prepared from three different garnet megablasts (up to $15-20 \mathrm{~cm}$ in size). Zircon crystals are included in garnet and kyanite and range in size from $\sim 10$ to $200 \mu \mathrm{m}$; they are either single, isolated crystals or clusters of two or more inclusions. The shape of the inclusions varies from rounded to elongated and almost idiomorphic with sharp corners and edges. Some of the zircon crystals also host mineral inclusions such as rutile, coesite, sheet silicates, and apatite, in agreement with previous observations (e.g., Gautiez-Putallaz et al. 2016).

Previous results (Gebauer et al. 1997; Gautiez-Putallaz et al. 2016) suggested the existence of two main zircon generations that can coexist within the same crystal in these rocks. The first one records the crystallization of the Permian protolith $(\sim 275$ $\mathrm{Ma})$, while the second domain is Eocene in age $(35.1 \pm 0.8 \mathrm{Ma})$ and related to metamorphism during the Alpine subduction. Chopin (1984) was the first to constrain this metamorphic event at UHP conditions by finding coesite inclusions within garnet. Optically single crystals of coesite inclusions have been found also in this study, especially within the garnet neoblasts $\left(\mathrm{SiO}_{2}\right.$ saturated whiteschists). Within the garnet megablasts, rare coesite inclusions have been found only at the external rim. More recent studies have constrained the metamorphic peak conditions in the diamond stability field at about 4-4.3 GPa and $730-750{ }^{\circ} \mathrm{C}$ (e.g., Hermann 2003; Ferrando et al. 2009; Gautiez-Putallaz et al. 2016).

The selection of completely buried zircon inclusions follows the protocol given by Campomenosi et al. (2018) for elastic thermobarometry applications (i.e., small, similar in size, nonfractured, and isolated inclusions within the center of a nonfractured host). On the other hand, the selection of the exposed grains (about $60 \%$ of the total number of zircon inclusions present in the samples) was based on criteria such as variable size of the inclusion and of the surrounding intact host (the effective host), exposure degree of the inclusions (optically estimated in terms of the ratio between the exposed and buried inclusion surface) and presence of fractures or any other kind of discontinuity in both the host and the inclusion.

\section{ANALYTICAL METHODS}

Exposed zircon inclusions were investigated by CC imaging first and Raman spectroscopy later to obtain textural and structural information, and finally by LA-ICP-MS for the chemical composition.

CC images were acquired with a ZEISS EV050 scanning electron microscope at the Institute of Geological Sciences, University of Bern, at low vacuum conditions (18 Pa), $12 \mathrm{kV}$, a beam current of $100 \mathrm{~mA}$ and a working distance of $9.5 \mathrm{~mm}$. CC images were obtained from well-polished, uncoated samples. It has been demonstrated that $\mathrm{CC}$ images correlate exactly to cathodoluminescence images and result from the complex interaction between the electron beam, the positive ions generated by electron-gas interactions in the chamber, a biased detector, and the sample (Griffin 2000; Watt et al. 2000). Internal check in the Bern laboratory confirmed that $\mathrm{CC}$ images are identical to panchromatic cathodoluminescence images.

Raman measurements were collected with two different Horiba Jobin-Yvon spectrometers: a T64000 triple-monochromator system operating in a subtractive mode and an Explora_Plus single-monochromator spectrometer at the University of Hamburg and Genova, respectively. The first one was equipped with a symphony $\mathrm{LN}_{2}$-cooled CCD detector, $1800 \mathrm{gr} / \mathrm{mm}$ holographic gratings, an Olympus BH-41 optical microscope and a Coherent $\mathrm{Ar}^{+}$laser, whereas the second with a Peltier-cooled CCD detector, a $2400 \mathrm{gr} / \mathrm{mm}$ grating, an Olympus BH-41 optical microscope, and Nd:YAG solid state laser. In both cases, using a slit width of $100 \mu \mathrm{m}$, the spectral resolution was approximately $2 \mathrm{~cm}^{-1}$ (determined using the photoluminescence line of $\mathrm{Sm}: \mathrm{SrB}_{4} \mathrm{O}_{7}$ for the $\mathrm{T} 64000$ and a Ne lamp for the Explora_Plus spectrometers), and therefore apparatus corrections of the peak widths were not required (Nasdala et al. 2001). Sufficient acquisition times and a surface density of the laser power were used to avoid sample overheating during the Raman measurements (Zhong et al. 2019). The instrumental accuracy in the peak position determination was about $0.35 \mathrm{~cm}^{-1}$ for the $\mathrm{T} 64000$ spectrometer and about $0.55 \mathrm{~cm}^{-1}$ for the Explora_Plus. These values refer to the half of the spectral pixel-pixel distance, which results from several instrumental factors, including the CCD detector array, groove density of gratings, and excitation-light wavelength.

However, both the instruments were manually calibrated to the silicon Raman peak at $520.5 \mathrm{~cm}^{-1}$ at each measurement session, and the stability of the instrumental setup was double-checked measuring reference crystals every $4 \mathrm{~h}$. The resulting standard deviation in the determination of the silicon peak along different days of measurements was approximately $0.04 \mathrm{~cm}^{-1}$. Furthermore, a crosscheck of the two data set measured with the two different spectrometers has been performed on several zircon grains to verify their consistency. Finally, the lateral spatial resolution of the two instruments is approximately $1 \mu \mathrm{m}$ and the probed volume at the sample surface is of about $1 \mu \mathrm{m}$ cube.

The Origin Lab-Pro 2018 software package was used for data evaluation. The collected spectra were baseline corrected for the continuum luminescence background when necessary, temperature-reduced to account for the BoseEinstein occupation factor (Kuzmany 2009), and normalized to the acquisition time (7 s). Peak positions, FWHMs, and integrated intensities were determined from fits with pseudo-Voigt functions [PV $=(1-q) \cdot$ Lorentz $+q$. Gauss, $q$ is the weight coefficient]. The criterion for the maximum number of fitted peaks was $\Delta I<I / 2$, where $I$ and $\Delta I$ are the calculated magnitude and uncertainty of each peak intensity, respectively.

Trace element analyses were obtained with an ASI-RESOlution $193 \mathrm{~nm}$ laser system coupled to an Agilent 7900 quadrupole Inductively Coupled Plasma mass spectrometer (ICPMS) at the Institute of Geological Sciences, University of Bern. The laser was tuned to a repetition rate of $5 \mathrm{~Hz}$ and an energy output of $4 \mathrm{~mJ}$ (corresponding to an $\mathrm{HV}$ of about 26-27 kV). The ICPMS was tuned for maximum sensitivity and minimum production of molecular species, maintaining $\mathrm{ThO}^{+} / \mathrm{Th}^{+}$at $<0.2 \%$. The spot size on the zircon crystal was $20 \mu \mathrm{m}$. Analyses were standardized to glass NIST 612, and zircon 91500 was run as a secondary standard to monitor accuracy. Stoichiometric Si was employed as an internal standard for zircon $\left(\mathrm{SiO}_{2}: 31.6 \mathrm{wt} \%\right)$. Reproducibility and accuracy were within $10 \%$ or less across all analyzed elements. The data were reduced with the freeware Iolite (Paton et al. 2011) and its data reduction scheme for trace elements (Woodhead et al. 2007).

\section{RESULTS \\ Partially exposed zircon inclusions \\ Charge contrast imaging. $\mathrm{CC}$ imaging of 64 inclusions} from samples DM17-13 and DM17-49 (Martiniana locality) and 83 grains from samples DMG4-6 and DM17-35 (Gilba 
locality) shows large variability in zircon internal texture. By combining the data on various crystals, it was possible to recognize at least four major domains (Fig. 1): (1) a dark (i.e., low CC-emission) domain, commonly corresponding to the crystal cores, (2) an oscillatory-zoned domain (medium-high $\mathrm{CC}$ emission), which usually corresponds to entire crystals or the core of elongated crystals and are further subdivided into oscillatory-dark and oscillatory-bright domains based on CC emission, (3) a transition (or undefined) domain, whose appearance is between the darker and a brighter domains and without a defined internal texture, and (4) a bright domain (i.e., high CC-emission), which usually belongs to the external rim of the crystals. About $70 \%$ of zircon inclusions from the
Gilba locality show a more elongated shape with a typical oscillatory-zoned core surrounded by 1 or 2 thin brighter rims. On the other hand, about $80 \%$ zircon inclusions from the Martiniana locality present more sub-idiomorphic shapes and more homogeneous internal texture. Furthermore, as already reported by Gautiez-Putallaz et al. (2016) for zircon hosted in Si undersaturated whiteschists, some of the analyzed inclusions in Martiniana, where garnet is more abundant, display oscillatory dark domains.

Raman spectroscopy. Zircon has tetragonal symmetry with space group $I 4_{1} /$ amd. According to group-theory analysis, the optical phonons at the Brillouin-zone center of zircon are (Kroumova et al. 2010):
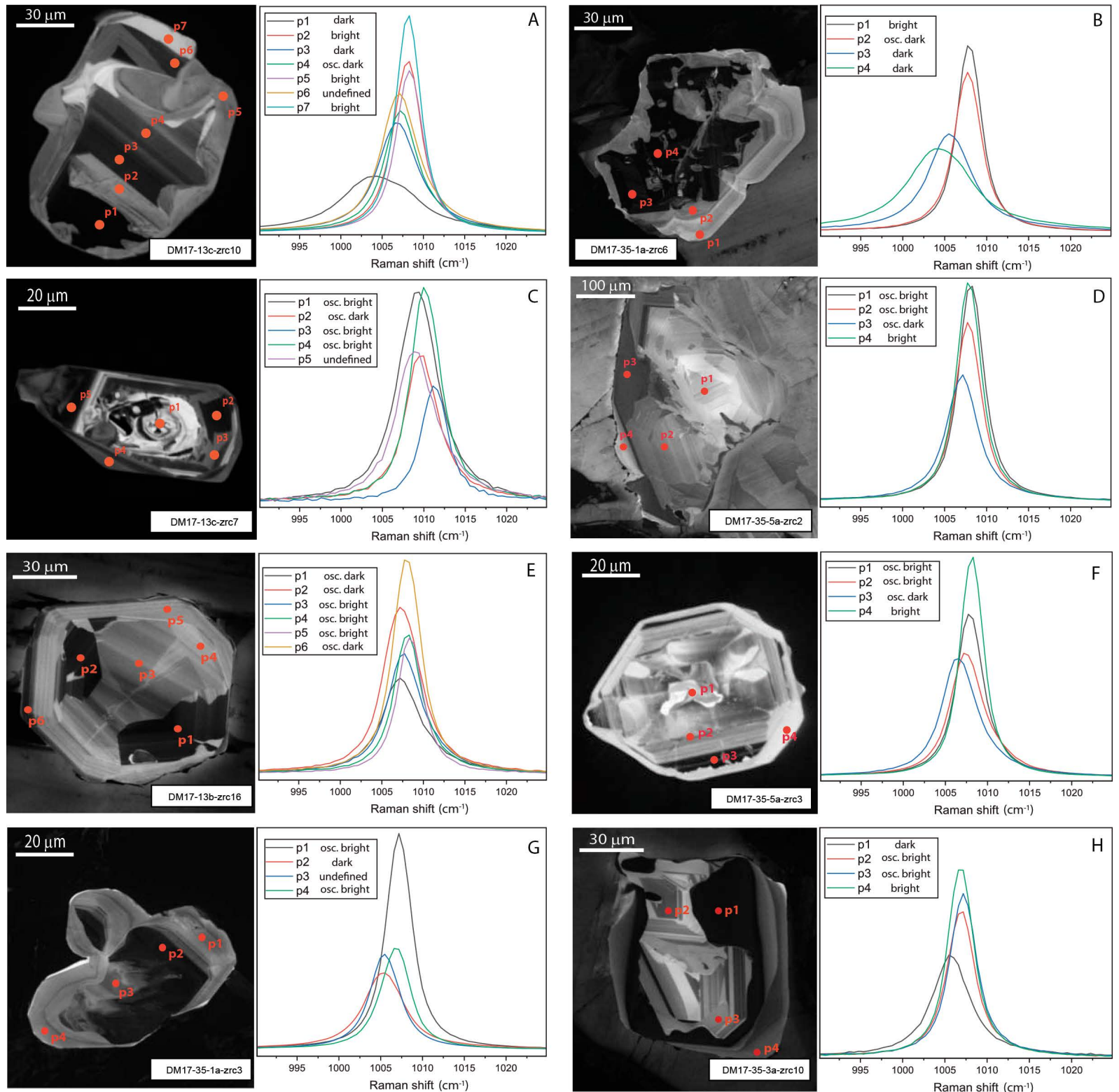

FigURE 1. Relationship between the $\mathrm{CC}$ emission domain and the corresponding Raman spectra $\left(\mathrm{B}_{1 \mathrm{~g}}\right.$ mode near $\left.1008 \mathrm{~cm}^{-1}\right)$ in partially exposed zircon inclusions. Samples DM17-35 are from Gilba locality while sample DM17-13 comes from the Martiniana locality. 
$\Gamma_{\text {opt }}=2 \mathrm{~A}_{1 \mathrm{~g}}+\mathrm{A}_{\mathrm{lu}}+\mathrm{A}_{2 \mathrm{~g}}+3 \mathrm{~A}_{2 \mathrm{u}}+4 \mathrm{~B}_{1 \mathrm{~g}}+\mathrm{B}_{\mathrm{lu}}+\mathrm{B}_{2 \mathrm{~g}}+2 \mathrm{~B}_{2 \mathrm{u}}+5 \mathrm{E}_{\mathrm{g}}+4 \mathrm{E}_{\mathrm{u}}$

The $A_{1 g}, B_{1 g}, B_{2 g}$, and $E_{g}$ modes are Raman-active and therefore a total of 12 Raman peaks can be observed in the spectrum of a randomly oriented zircon. According to previous studies (Knittle and Williams 1993; Nasdala et al. 2001; Binvignat et al. 2018), both pressure and metamictization affect most strongly the $\mathrm{B}_{1 \mathrm{~g}}$ mode near $1008 \mathrm{~cm}^{-1}$, originating from anti-symmetrical $\mathrm{SiO}_{4}$ stretching. For this reason, our discussion is mainly focused on this phonon mode.

The Raman spectroscopic analysis is based on 250 spectra collected from 60 zircon inclusions in different $\mathrm{CC}$ domains. As an example, Figure 1 shows the variation of the internal $\mathrm{SiO}_{4}$ antisymmetric stretching mode $\left(\mathrm{B}_{1 \mathrm{~g}}\right)$ near to $1008 \mathrm{~cm}^{-1}$ of representative zircon inclusions exhibiting heterogeneous $\mathrm{CC}$ emission. Generally, dark CC domains correspond to Raman peak broadening and shift toward lower wavenumbers when compared to those collected across brighter or oscillatoryzoned domains. Furthermore, transition CC domains show transitional average features also for the Raman spectra.

A more quantitative evaluation of the structural state of zircon crystals can be done plotting the relationship between the phonon wavenumber $\omega$ and the corresponding peak widht $\Gamma$ (Geisler and Pidgeon 2002; Zhong et al. 2019). The data measured on partially exposed zircon inclusions (Fig. 2) show two different trends that correlate with the four different zircon domains (bright, oscillatory-zoned, undefined, and dark).

The first trend is characterized by $\omega<1008 \mathrm{~cm}^{-1}, 4.5 \mathrm{~cm}^{-1}$ $\leq \Gamma<12 \mathrm{~cm}^{-1}$, and $\omega(\Gamma)$ with a negative slope (inverse correlation). The data points on this trend mostly correspond to zircon domains with dark and oscillatory-dark CC emission.

The second trend, at higher wavenumbers, is characterized by $1008 \mathrm{~cm}^{-1}<\omega<1011 \mathrm{~cm}^{-1}, 3.5 \mathrm{~cm}^{-1}<\Gamma<5.5 \mathrm{~cm}^{-1}$, and $\omega(\Gamma) \approx$ constant. This trend is mostly defined by zircon domains with bright and oscillatory(-bright) $\mathrm{CC}$ emission.

Note that, on average, oscillatory-dark domains display the largest $\Gamma$ and lowest wavenumber with respect to oscillatorybright domains. On the other hand, undefined domains are difficult to classify since they scatter over the entire data.

LA-ICP-MS. The different types of zircon domains also show differences in trace-element composition. Tables $1 \mathrm{a}-1 \mathrm{~b}$ report representative major, minor, and trace elements composition of analyzed zircon crystal inclusions as determined

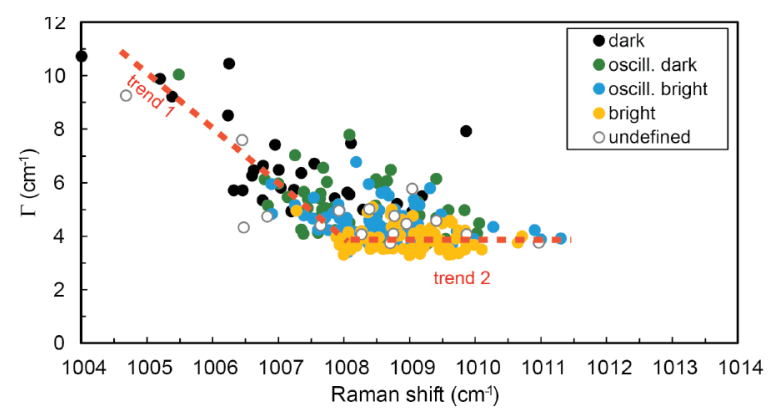

FIGURE 2. $\Gamma$ vs. Raman shift of the antisymmetric $\mathrm{SiO}_{4}$ stretching $\mathrm{B}_{1 \mathrm{~g}}$ mode near $1008 \mathrm{~cm}^{-1}$ of partially exposed crystals with highlighted CC-emission domains. by LA-ICP-MS with the associated CC-emission domains. Hf concentration is usually around $11000 \mu \mathrm{g} / \mathrm{g}$ and only few analyses show higher values up to $14000 \mu \mathrm{g} / \mathrm{g}$. U content varies significantly between oscillatory-bright zoned, oscillatorydark, and dark CC domains, with the last showing, usually, the highest concentration (see for example DM17-35-3a-zrc10-p1 and p2 or even DM17-35-3a-zrc14-p3 and p2 in Table 1a). U contents vary from a few hundred $\mu \mathrm{g} / \mathrm{g}$ in the bright domains to $5000 \mu \mathrm{g} / \mathrm{g}$ for the darker domains and generally show an inverse relationship with $\mathrm{CC}$ emission, indicating that $\mathrm{U}$ suppresses luminescence (e.g., Rubatto and Gebauer 2000).

In line with previous REE data sets (e.g., Gautiez-Putallaz et al. 2016), zircon cores with oscillatory or convolute zoning have a steep HREE-enriched pattern with a pronounced negative Eu anomaly (e.g., Fig. 3c p1). This REE pattern is characteristic of conditions where plagioclase was present and garnet was absent, i.e., during the crystallization of the granitic protholith in Permian times (e.g., Gautiez-Putallaz et al. 2016).

The rims and many crystals with oscillatory zoning have lower REE contents and a flat HREE pattern with no or weak Eu-anomaly (Fig. 3). This REE pattern is diagnostic for zircons formed during prograde to peak metamorphism, where feldspar is no longer stable (e.g., Gautiez-Putallaz et al. 2016). The variable slope of the HREE patterns ranging from slightly positive to even negative (Fig. 3c) indicates zircon growth together with garnet, as both minerals compete for the HREE. Therefore a first distinction between pre-Alpine inherited igneous zircon and Alpine metamorphic zircon can be obtained from the trace element analysis. Notably, several large crystals that display a fine oscillatory to sector zoning more or less surrounded by a dark or light rim present a depletion in HREE across the entire grain (e.g., Figs. 3d and 3e). This demonstrates that oscillatory zoning occurs also in Alpine metamorphic zircon and is not restricted to inherited igneous zircon, demonstrating that both $\mathrm{CC}$ and trace elements are required for the correct classification of the zircon.

Previous analyses on the same locality demonstrated that inherited zircon cores are ca. $275 \mathrm{Ma}$ old and metamorphic rims are $35.1 \pm 0.8 \mathrm{Ma}$ old (Gebauer et al. 1997; GauthiezPutallaz et al. 2016). In our case, a rough estimate of the age can also be obtained from the LA-ICP-MS data, even if proper age standardization and common $\mathrm{Pb}$ correction have not been performed. The ${ }^{206} \mathrm{~Pb} /{ }^{238} \mathrm{U}$ values estimated in such a way are in general agreement with those obtained before on zircon from the same locality and correlate with the distinction made from the REE patterns (Gauthiez-Putallaz et al. 2016). This rough age estimate allows distinguishing between Alpine domains (usually lower U content and bright or oscillatory-bright CC domains) and pre-Alpine domains (higher U content and dark or oscillatory-zoned CC domains at the zircon cores) especially in cases where REE patterns might be ambiguous (see Table 2). Notably the large crystals with oscillatory-sector zoning and low HREE content are of Alpine age.

Assuming an age of $275 \mathrm{Ma}$ for the pre-Alpine and $35 \mathrm{Ma}$ for the Alpine domains (Gebauer et al. 1997; Gauthiez-Putallaz et al. 2016) and based on the measured U-Th composition of the zircon domains, their accumulation radiation doses $D_{a}$ was calculated as follows (e.g., Nasdala et al. 2001): 
TABLE 1a. Major, minor, and trace elements (in $\mu \mathrm{g} / \mathrm{g}$ ) of zircon inclusions (spots are referred to Fig. 3)

\begin{tabular}{|c|c|c|c|c|c|c|c|c|}
\hline Sample name & CC domain & $\mathrm{Ti}$ & $\bar{Y}$ & $\mathrm{Nb}$ & $\mathrm{Hf}$ & $\mathrm{Pb}$ & Th & $\mathrm{U}$ \\
\hline DM17-35-3A-zrc8-p1 & osc. dark & 2.28 & 1639 & 3.16 & 10300 & 154 & 158 & 1436 \\
\hline DM17-35-3A-zrc8-p2 & osc. bright & 4.70 & 2651 & 8.73 & 7710 & 390 & 793 & 1241 \\
\hline DM17-35-3A-zrc10-p1 & osc. bright & 35.3 & 492 & 34.2 & 10880 & 10.0 & 84.9 & 378 \\
\hline DM17-35-3A-zrc10-p2 & dark & 5.03 & 197 & 5.98 & 13690 & 52.4 & 84.9 & 378 \\
\hline DM17-35-3A-zrc10-p3 & bright & 7.90 & 183 & 4.01 & 10840 & 24.3 & 6.85 & 883 \\
\hline DM17-35-3A-zrc14-p1 & osc. bright & 2.91 & 850 & 1.94 & 12450 & 138 & 40.7 & 730 \\
\hline DM17-35-3A-zrc14-p2 & osc. dark & 6.90 & 3140 & 8.05 & 10300 & 326 & 696 & 1900 \\
\hline DM17-35-3A-zrc14-p3 & osc. bright & 4.90 & 1820 & 4.50 & 11660 & 237 & 158 & 1320 \\
\hline DM17-35-3A-zrc14-p4 & osc. bright & 2.67 & 1353 & 1.42 & 8680 & 66.1 & 73.4 & 333 \\
\hline DM17-35-3A-zrc18-p1 & osc. bright & 6.85 & 1850 & 1.64 & 9100 & 308 & 179.6 & 474 \\
\hline DM17-35-3A-zrc13-p2 & osc. bright & 1.69 & 920 & 3.05 & 9570 & 130 & 89.0 & 673 \\
\hline DM17-35-3A-zrc13-p1 & osc. bright & 1.79 & 1051 & 2.80 & 10960 & 154 & 87.0 & 828 \\
\hline DM17-35-3A-zrc13-p3 & bright & 3.04 & 1150 & 4.26 & 7070 & 242 & 336 & 635 \\
\hline DM17-35-5A-zrc2-p1 & osc. bright & 10.3 & 211 & 5.86 & 11770 & 9.65 & 22.4 & 367 \\
\hline DM17-35-5A-zrc2-p2 & osc. bright & 8.81 & 83.3 & 9.95 & 11190 & 17.6 & 34.3 & 695 \\
\hline DM17-35-5A-zrc2-p3 & osc. dark & 8.30 & 83.4 & 8.75 & 11270 & 31.4 & 34.9 & 1208 \\
\hline DM17-35-5A-zrc2-p4 & osc. dark & 4.60 & 58.5 & 3.10 & 11030 & 32.3 & 11.3 & 1148 \\
\hline DM17-35-5A-zrc2-p5 & bright & 5.10 & 198 & 2.85 & 11170 & 28.6 & 7.17 & 1092 \\
\hline DM17-35-5A-zrc3-p1 & osc. bright & 2.02 & 930 & 0.76 & 9950 & 51.5 & 44.1 & 253 \\
\hline DM17-35-5A-zrc14-p1 & osc. bright & 48.3 & 1682 & 26.8 & 9768 & 8.94 & 87.1 & 380 \\
\hline DM17-35-5A-zrc14-p2 & dark & 10.1 & 490 & 5.49 & 11520 & 16.4 & 15.7 & 530 \\
\hline DM17-35-5A-zrc14-p3 & bright & 10.8 & 430 & 6.06 & 10840 & 3.80 & 12.3 & 112 \\
\hline DM17-35-5A-zrc14-p4 & bright & 22.5 & 632 & 20.1 & 11400 & 8.96 & 32.7 & 370 \\
\hline DM17-35-5A-zrc14-p5 & osc. dark & 7.10 & 262 & 6.38 & 12790 & 10.5 & 8.84 & 407 \\
\hline DM17-35-1a-zrc6-p2 & osc. dark & 14.3 & 720 & 8.31 & 14570 & 65.8 & 41.1 & 3147 \\
\hline DM17-35-1a-zrc6-p3 & dark & 20.2 & 826 & 7.62 & 12610 & 77.1 & 32.8 & 2949 \\
\hline DM17-35-1a-zrc3-p1 & osc. bright & 13.3 & 204 & 1.63 & 12930 & 19.7 & 9.89 & 452 \\
\hline DM17-35-1a-zrc3-p2 & dark & 9.90 & 103.3 & 2.98 & 11690 & 73.7 & 7.82 & 3154 \\
\hline DM17-35-1a-zrc3-p3 & osc. bright & 13.1 & 161.8 & 3.09 & 12280 & 80.7 & 12.1 & 3700 \\
\hline DM17-35-1a-zrc9-p1 & osc. bright & 6.38 & 179.3 & 2.47 & 12190 & 43.7 & 16.8 & 1640 \\
\hline DM17-35-1a-zrc10-p1 & osc. bright & 10.8 & 587 & 2.42 & 11220 & 9.10 & 10.7 & 249.8 \\
\hline DM17-35-1a-zrc10-p2 & osc. bright & 790 & 536 & 8.53 & 10980 & 8.71 & 13.0 & 261 \\
\hline DM17-13-b-zrc13-p1 & osc. bright & 17.0 & 1479 & 21.6 & 11960 & 31.1 & 51.5 & 1307 \\
\hline DM17-13-b-zrc13-p2 & osc. bright & 11.8 & 1209 & 11.5 & 10820 & 22.5 & 26.6 & 755 \\
\hline DM17-13-b-zrc15-p2 & osc. bright & 6.30 & 649 & 4.80 & 10180 & 16.9 & 11.5 & 604 \\
\hline DM17-13-b-zrc15-p3 & osc. bright & 6.10 & 589 & 5.86 & 10310 & 9.37 & 7.11 & 346 \\
\hline DM17-13-b-zrc16-p1 & osc. bright & 16.8 & 1817 & 13.7 & 10840 & 34.7 & 35.2 & 1342 \\
\hline DM17-13-b-zrc16-p2 & osc. bright & 8.50 & 383 & 3.96 & 11030 & 16.8 & 14.9 & 562 \\
\hline DM17-13-b-zrc16-p3 & osc. dark & 20.9 & 1504 & 29.9 & 10550 & 43.3 & 56.8 & 1538 \\
\hline DM17-13-b-zrc16-p4 & osc. bright & 5.66 & 227.8 & 4.54 & 11550 & 10.3 & 18.5 & 435.3 \\
\hline DM17-13-c-zrc7-p1 & osc. bright & 16.5 & 2140 & 2.44 & 10600 & 125 & 149 & 719 \\
\hline DM17-13-c-zrc7-p2 & osc. dark & 10.2 & 1790 & 7.65 & 11900 & 21.1 & 13.2 & 950 \\
\hline DM17-13-c-zrc7-p3 & undefined & 10.1 & 1982 & 12.5 & 12280 & 25.4 & 17.8 & 1037 \\
\hline DM17-13-c-zrc4-p1 & bright & 27.0 & 3250 & 24.3 & 12380 & 32.4 & 35.2 & 1335 \\
\hline DM17-13-c-zrc4-p2 & undefined & 11.8 & 2100 & 13.6 & 11850 & 56.1 & 39.8 & 931 \\
\hline DM17-13-c-zrc10-p1 & dark & 20.9 & 2680 & 33.7 & 11080 & 102 & 33.0 & 5060 \\
\hline DM17-13-c-zrc10-p2 & bright & 7.30 & 582 & 4.56 & 11860 & 28.0 & 7.63 & 1175 \\
\hline DM17-13-c-zrc10-p3 & osc. dark & 10.7 & 1540 & 8.24 & 11480 & 46.2 & 6.67 & 2004 \\
\hline
\end{tabular}

Note: Precision plus accuracy of measurements is within $10 \%$.
$\Gamma$ mostly between 3.5 and $5.5 \mathrm{~cm}^{-1}$. Only a few analyses reach values up to $6.5 \mathrm{~cm}^{-1}$ (Fig. 4). The overlap between the two sets of inclusions is limited to about $10 \%$ of the analyses.

The data from the exposed and fully enclosed zircon inclusions can also be analyzed in terms of the wavenumber difference $(\Delta \omega)$ between the $\mathrm{B}_{\mathrm{lg}}$ mode near $1008 \mathrm{~cm}^{-1}$ and the $\mathrm{A}_{1 \mathrm{~g}}$ mode near $440 \mathrm{~cm}^{-1}$ rather than the absolute wavenumber. In this way, since the data were collected along different sessions of measurements, we can avoid eventual effects due to instrumental drift. As shown previously by Knittle and Williams (1993) and more recently by Binvignat et al. (2018) both phonon modes show an increase in the phonon wavenumber as the hydrostatic pressure increases. However, as reported from the same authors, the $A_{1 g}$ mode shows a weaker pressure dependence with respect to the $\mathrm{B}_{\mathrm{lg}}$ mode and, therefore, to an increase of the pressure acting on the crystal should correspond an increase in the value of $\Delta \omega$ (Fig. 5).

\section{Discussion}

\section{Effect of minor and trace elements on zircon Raman shift}

A large number of non-formula elements can be incorporated in the zircon crystal structure, however, most of them are usually far below $1 \mathrm{wt} \%$ (e.g., Hoskin and Schaltegger 2003), with the notable exception of

$$
D_{\alpha}=\frac{0.9928 N_{A} c_{\mathrm{U}} n_{238}}{10^{6} m_{238}}\left(e^{\lambda_{238} t}-1\right)+\frac{0.0072 N_{A} c_{\mathrm{U}} n_{235}}{10^{6} m_{235}}\left(e^{\lambda_{235} t}-1\right)+.
$$

$\frac{N_{A} c_{\mathrm{Th}} n_{232}}{10^{6} m_{232}}\left(e^{\lambda_{232} t}-1\right)$

where $\lambda_{\mathrm{n}}$ and $m_{\mathrm{n}}$ are, respectively, the nuclear decay constant and mass of the corresponding isotope $\left({ }^{238} \mathrm{U},{ }^{235} \mathrm{U}\right.$, and ${ }^{232} \mathrm{Th}$ ) (Steiger and Jaeger 1977), the coefficient $n$ represents the number of $\alpha$ decays per nucleus $\left(n_{238}=8, n_{235}=7\right.$, and $\left.n_{232}=6\right)$ and $N_{A}$ is the Avogadro's number $c_{\mathrm{U}}$ and $c_{\mathrm{Th}}$ are the measured concentrations of $U$ and $\mathrm{Th}$, respectively, and $t$ the time. Note that Equation 1 presumes a $\mathrm{U}$ isotopic composition of $99.28 \%{ }^{238} \mathrm{U}$ and $0.72 \%$ ${ }^{235} \mathrm{U}$. The resulting $D_{\mathrm{a}}$ values are reported in Table 2.

\section{Completely buried zircon inclusions}

The Raman spectra collected on the completely buried zircon crystals have higher wavenumbers (from $1010 \mathrm{~cm}^{-1}$ up to $1013.5 \mathrm{~cm}^{-1}$ ) with respect to the partially exposed grains, with a
Hf. Raman data measured on isomorphic series $\mathrm{ZrSiO}_{4}-\mathrm{HfSiO}_{4}$, suggest that even if as much as $25 \%$ of all $\mathrm{Zr}$ ions are replaced by $\mathrm{Hf}$ the frequency variation of the main peaks does not exceed $3 \mathrm{~cm}^{-1}$ (Hoskin and Rodgers 1996). Furthermore, Nasdala et al. (2002) suggested that positions and $\Gamma$ of the main peaks in annealed, metamict gemstone-quality zircon, containing up to $6000 \mu \mathrm{g} / \mathrm{g}$ of $\mathrm{U}$ and $16300 \mu \mathrm{g} / \mathrm{g}$ of Hf, deviate less than $1 \mathrm{~cm}^{-1}$ from the data of pure well-crystalline $\mathrm{ZrSiO}_{4}$. Therefore, since our exposed zircon inclusions contain impurities and trace elements below these values (maximum $\mathrm{U}$ content is $5060 \mu \mathrm{g} / \mathrm{g}$ and maximum Hf content is $14570 \mu \mathrm{g} / \mathrm{g}$, see Table 1 ), the effect of chemical variations on zircon Raman spectra is negligible.

Zircon variation in HREE composition (i.e., depletion in the Alpine domains) has been previously interpreted as the result of growth zoning during metamorphism in a fractionating bulk composition where REE are largely incorporated in garnet (e.g., Gautiez-Putallaz et al. 2016). Indeed, the garnet host shows a similar REE pattern along a core-to-rim line profile (e.g., 
TABLE 1b. REE composition (in $\mu \mathrm{g} / \mathrm{g}$ ) of zircon inclusions (spots are referred to Fig. 3)

\begin{tabular}{|c|c|c|c|c|c|c|c|c|c|c|c|c|c|c|c|}
\hline Sample name & CC domain & $\mathrm{La}$ & $\mathrm{Ce}$ & $\mathrm{Pr}$ & $\mathrm{Nd}$ & $\mathrm{Sm}$ & $\mathrm{Eu}$ & $\mathrm{Gd}$ & $\mathrm{Tb}$ & Dy & $\mathrm{Ho}$ & Er & $\mathrm{Tm}$ & $\mathrm{Yb}$ & $\overline{L u}$ \\
\hline DM17-35-3A-zrc8-p1 & Osc. dark & 0.04 & 5.90 & 0.21 & 2.65 & 5.07 & 0.15 & 28.9 & 10.5 & 143 & 54.9 & 273 & 60.7 & 553 & 112 \\
\hline DM17-35-3A-zrc8-p2 & Osc. bright & 0.46 & 18.7 & 0.40 & 6.43 & 12.1 & 0.26 & 65.8 & 21.8 & 267 & 91.0 & 411 & 79.6 & 653 & 128 \\
\hline DM17-35-3A-zrc10-p1 & Osc. bright & b.d.l. & 2.80 & 0.04 & 1.06 & 6.80 & 2.23 & 70.5 & 16.0 & 86.2 & 12.3 & 29.5 & 4.80 & 38.9 & 6.95 \\
\hline DM17-35-3A-zrc10-p2 & dark & b.d.l. & 0.59 & b.d.I. & b.d.l. & 0.51 & 0.11 & 4.78 & 2.01 & 20.1 & 4.93 & 15.8 & 2.73 & 22.2 & 3.98 \\
\hline DM17-35-3A-zrc10-p3 & bright & b.d.l. & 1.03 & b.d.l. & 0.14 & 0.54 & 0.14 & 4.54 & 1.83 & 19.6 & 4.77 & 17.0 & 2.67 & 19.3 & 2.80 \\
\hline DM17-35-3A-zrc14-p1 & Osc. bright & b.d.l. & 0.44 & b.d.I. & 0.17 & 1.06 & 0.19 & 10.05 & 4.41 & 63.0 & 26.4 & 143 & 35.7 & 351 & 73.0 \\
\hline DM17-35-3A-zrc14-p2 & Osc. dark & 12.0 & 37.0 & 3.10 & 14.6 & 11.5 & 0.55 & 61.0 & 22.7 & 291 & 104 & 497 & 101 & 839 & 156 \\
\hline DM17-35-3A-zrc14-p3 & Osc.bright & 0.49 & 3.40 & 0.21 & 1.19 & 3.14 & 0.19 & 22.0 & 9.90 & 142 & 57.1 & 305 & 71.7 & 670 & 135 \\
\hline DM17-35-3A-zrc14-p4 & Osc. bright & 0.04 & 1.54 & 0.04 & 1.13 & 3.51 & 0.15 & 20.8 & 8.45 & 115 & 44.7 & 219 & 46.1 & 408 & 79.5 \\
\hline DM17-35-3A-zrc18-p1 & Osc. bright & 1.12 & 5.21 & 0.85 & 8.20 & 10.7 & 0.97 & 51.6 & 16.9 & 192 & 62.1 & 269 & 53.6 & 454 & 84.8 \\
\hline DM17-35-3A-zrc13-p2 & Osc. bright & b.d.l. & 2.78 & b.d.l. & 0.29 & 1.49 & 0.12 & 9.77 & 4.76 & 73.3 & 30.2 & 161 & 37.3 & 350 & 71.9 \\
\hline$c 13-p 1$ & Osc. bright & b.d.l. & 2.38 & 0.02 & 0.33 & 1.08 & 0.10 & 9.02 & 4.55 & 75.7 & 32.9 & 182 & 43.8 & 424 & 88.1 \\
\hline DM17-35-3A-zrc13-p3 & bright & 0.34 & 14.1 & 0.17 & 1.78 & 3.15 & 0.73 & 16.5 & 6.16 & 85.0 & 34.4 & 185 & 44.1 & 442 & 97.0 \\
\hline DM17-35-5A-zrc2-p1 & Osc. bright & 0.08 & 1.08 & 0.04 & 0.42 & 3.30 & 1.36 & 53.7 & 10.8 & 48.2 & 5.31 & 11.1 & 1.39 & 10.0 & 1.69 \\
\hline $2-p 2$ & Osc. bright & b.d.l. & 0.75 & b.d.l. & 0.34 & 4.74 & 1.41 & 43.7 & 6.35 & 22.2 & 2.26 & 4.55 & 0.61 & 5.21 & 0.90 \\
\hline DM1 & Osc. dark & b.d.l. & 0.99 & b.d.I. & 0.12 & 3.47 & 1.13 & 34.8 & 5.44 & 19.9 & 2.26 & 4.60 & 0.61 & 5.82 & 1.03 \\
\hline 2-p4 & Osc. $c$ & b.d.l. & 0.57 & b.d.l. & b.d.l. & 0.91 & 0.37 & 13.3 & 2.64 & 12.1 & 1.45 & 3.48 & 0.61 & 4.62 & 0.88 \\
\hline$-\mathrm{p} 5$ & brig & b.d.l. & 0.81 & b.d.I. & b.d.l. & 0.62 & 0.14 & 5.83 & 1.92 & 20.3 & 5.27 & 18.7 & 3.33 & 23.5 & 3.37 \\
\hline DM1 & Osc. bright & 0.06 & 1.27 & 0.11 & 0.78 & 2.50 & 0.07 & 14.1 & 5.45 & 76.9 & 30.7 & 154 & 33.6 & 301 & 59.3 \\
\hline 4-p1 & Osc. b & b.d.l. & 3.06 & 0.02 & $1.1 \mathrm{c}$ & 10.1 & 3.72 & 164 & 46.6 & 288 & 47.8 & 117 & 16.7 & 118 & 19.3 \\
\hline 14-p2 & dark & 0.40 & 2.11 & 0.19 & 1.35 & 2.51 & 0.86 & 31.0 & 9.74 & 70.3 & 12.9 & 32.5 & 4.76 & 31.7 & 5.42 \\
\hline 4-p3 & bri & b.d.l. & 0.75 & b.d.I. & 0.2 & 2.44 & 0.65 & 31.7 & 9.81 & 68.9 & 11.6 & 30.1 & 4.66 & 4.8 & 6.21 \\
\hline 4-p4 & bright & b.d.l. & 1.89 & 0.04 & 0.68 & 4.14 & 1.39 & 54.2 & 15.9 & 104 & 16.4 & 40.5 & 5.53 & 38.8 & 6.39 \\
\hline DM17-35-5A-zrc14-p5 & Osc. dark & b.d.l. & 0.72 & b.d.l. & 0.10 & 1.19 & 0.40 & 16.3 & 5.58 & 38.4 & 6.47 & 16.4 & 2.28 & 15.6 & 2.60 \\
\hline 6-p2 & Osc. dark & b.d.l. & 1.42 & 0.02 & 0.66 & 6.17 & 1.75 & 64.7 & 18.2 & 118 & 18.4 & 43.4 & 5.31 & 0.5 & 4.17 \\
\hline -p3 & dark & 0.08 & 2.06 & 0.07 & 1.0 & 7.30 & 1.84 & 72.4 & 20.2 & 131 & 21.2 & 49.2 & 6.34 & 36.5 & 5.05 \\
\hline & Osc. b & 0.06 & 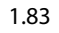 & 0.06 & 08 & 1.35 & 0.27 & 8.60 & 24 & 21.8 & & & 336 & & 3.52 \\
\hline -p2 & darl & b.d.l. & 0.45 & b.d.l. & 0.1 & 0.74 & 0.23 & 7.75 & 2.34 & 15.4 & 2.60 & 7.27 & 1.19 & 8.46 & 1.46 \\
\hline DM17-35-1a-z & Osc. bright & b.d.l. & 0.95 & 0.02 & 0.26 & 1.50 & 0.44 & 14.4 & 4.10 & 25.8 & 4.42 & 12.0 & 2.04 & 14.0 & 2.42 \\
\hline & Osc. & b.d.l. & 0.53 & b.d.l. & 0.1 & 1.40 & 0.46 & 16.1 & 4.63 & 27.3 & 4.51 & 12.9 & 2.03 & 14.5 & 2.48 \\
\hline$-p 1$ & Osc. & b.d.l. & 0.56 & b.d.I. & 0.2 & 3.06 & 0.93 & 38.1 & 11.7 & 89.1 & 18.0 & 51.8 & 7.30 & 47.6 & 7.10 \\
\hline -p2 & Osc. & 0.05 & 0.70 & b.d.I. & 0.20 & 2.45 & 0.91 & 36.0 & 10.4 & 81.6 & 16.8 & 47.4 & 6.71 & 42.7 & 6.59 \\
\hline & Osc. & b.d.l. & 1.14 & 0.01 & 0.4 & 7.78 & 3.36 & 110 & 32.1 & 219 & 42.4 & 115 & 16.5 & 96.9 & 14.3 \\
\hline 3-p2 & Osc. & b.d.l. & 0.64 & b.d.I. & 0.4 & 5.57 & 2.46 & 86.6 & 25.6 & 177 & 35.2 & 95.6 & 14.4 & 85.9 & 12.7 \\
\hline & & b.d.l. & 0.67 & b.d.I. & 0.1 & 2.26 & 1.08 & 39.2 & 11.2 & 90.3 & 18.9 & 58.5 & 9.16 & 62.8 & 10.9 \\
\hline & & & & $b d$, & & 1.8 & 0.97 & 33 & 10 & 80.4 & 17.2 & 54.2 & 8.52 & 55.6 & 8.56 \\
\hline 3-b-zrc16-p1 & Osc. bright & b.d.l. & 0.88 & b.d.I. & 0.58 & 9.34 & 4.24 & 147 & 40.6 & 288 & 54.3 & 149 & 21.4 & 133 & 19.4 \\
\hline & & & 0.41 & & 0.16 & 2.34 & 0.96 & 32.0 & 8.26 & 58.5 & 11.0 & 30.3 & 4.72 & 33.8 & 6.03 \\
\hline 5-p3 & 0 & b.c & 1.2. & b.d.I. & 0. & 7.11 & 3.05 & 116 & 33.2 & 233 & 44.0 & 116 & 16.2 & 99.6 & 13.2 \\
\hline DM & Osc. & b.d.l. & 0.35 & b.d.I. & 0.1 & 1.32 & 0.54 & 15.3 & 4.54 & 32.1 & 6.00 & 16.2 & 3.04 & 26.4 & 5.43 \\
\hline & & & & & & & & 31 & 13 & & 69.7 & 334 & 71.6 & 47 & 122 \\
\hline DM17-13-c-zrc7-p2 & Osci. dark & b.d.l. & 0.57 & b.d.I. & 0.15 & 2.20 & 1.22 & 54.0 & 23.0 & 230 & 55.0 & 164 & 25.0 & 160 & 24.0 \\
\hline 13-c-zrc7-p3 & undefined & b.d.l. & 0.73 & b.d.l. & b.d.l. & 3.23 & 1.36 & 54.2 & 24.5 & 250 & 63.5 & 197 & 30.1 & 210 & 32.9 \\
\hline & & & 1.6 & & 0.53 & 6.90 & 2.72 & 97.7 & 39.0 & & 104 & 335 & 54.3 & 407 & 69.1 \\
\hline 13-c-zrc4-p2 & undefined & b.d.l. & 1.21 & b.d.l. & 0.28 & 3.64 & 1.53 & 54.4 & 21.5 & 230 & 67.1 & 247 & 45.7 & 377 & 68.5 \\
\hline DM17-13-c-zrc10-p1 & & & 1.97 & b.d.I. & 0.4 & 6.10 & 2.69 & 90.3 & 33.8 & 310 & 84.0 & 326 & 64.0 & 536 & 94.8 \\
\hline DM1 & brig & bd & 0.3 & b.d.l. & b.d & 0.95 & 0.44 & 18.9 & 7.34 & 69.3 & 19.0 & 74.4 & 14.6 & 126 & 23.0 \\
\hline DM17-13-c-zrc10-p3 & Osc. dark & b.d.l. & 0.62 & b.d.I. & 0.32 & 2.79 & 1.19 & 40.9 & 16.8 & 170 & 47.9 & 191 & 39.1 & 347 & 65.0 \\
\hline
\end{tabular}

Note: Precision plus accuracy of measurements is within $10 \%$.

Gautiez-Putallaz et al. 2016), indicating equilibrium conditions with the associated zircon grains. The qualitative ${ }^{206} \mathrm{~Pb} /{ }^{238} \mathrm{U}$ measurements confirm that zircon crystals showing a flat HREE pattern throughout the entire grain are completely metamorphic in origin. In this regard, it is important to note that, although REE chemical zonation does not influence the main Raman scattering features, such as peak broadening and position, it can give rise to heterogeneous photoluminescence, and hence to different background levels of the Raman spectra collected from zones. Besides, depending on the excitation laser wavelength, additional photoluminescence peaks may be observed next to the fundamental Raman peaks of zircon.

\section{Metamorphic vs. inherited zircon domains}

Charge contrast or cathodoluminesce imaging of metamorphic and inherited zircons usually show notable differences in terms of the corresponding internal texture (e.g., Rubatto and Gebauer 2000; Corfu et al. 2003). Oscillatory-zoned grains have been chemically interpreted as the result of alternating deple- tion and enrichment in trace elements (i.e., $\mathrm{U}$ and $\mathrm{Y}$ ) during crystal growth at the crystal-melt interface. On the other hand, bright domains at the rim of the crystals, usually show higher and homogeneous CL-emission, presenting irregular shape that often overgrowths the pre-existing crystals whose texture can sometime be evident as a relict (Rubatto and Gebauer 2000). Our results indicate that metamorphic zircon can also include dark, bright, oscillatory, and undefined CC domains. This observation warns against using internal zircon zoning alone for distinguishing magmatic vs. metamorphic zircon.

As portrayed in Figure 2, bright and oscillatory-bright domains (yellow and blue spots, respectively), have the same Raman spectral features and define the "non-metamict" domain in the diagram (see details below). Therefore, despite their magmatic or metamorphic orgin, from a structural point of view, these two domains are effectively equivalent and there will be no difference in the calculated residual stress even if the two domains coexist within the same crystal.

Finally, if we plot our data in a $\Gamma$ of the $B_{1 \mathrm{~g}}$ mode near $1008 \mathrm{~cm}^{-1}$ 

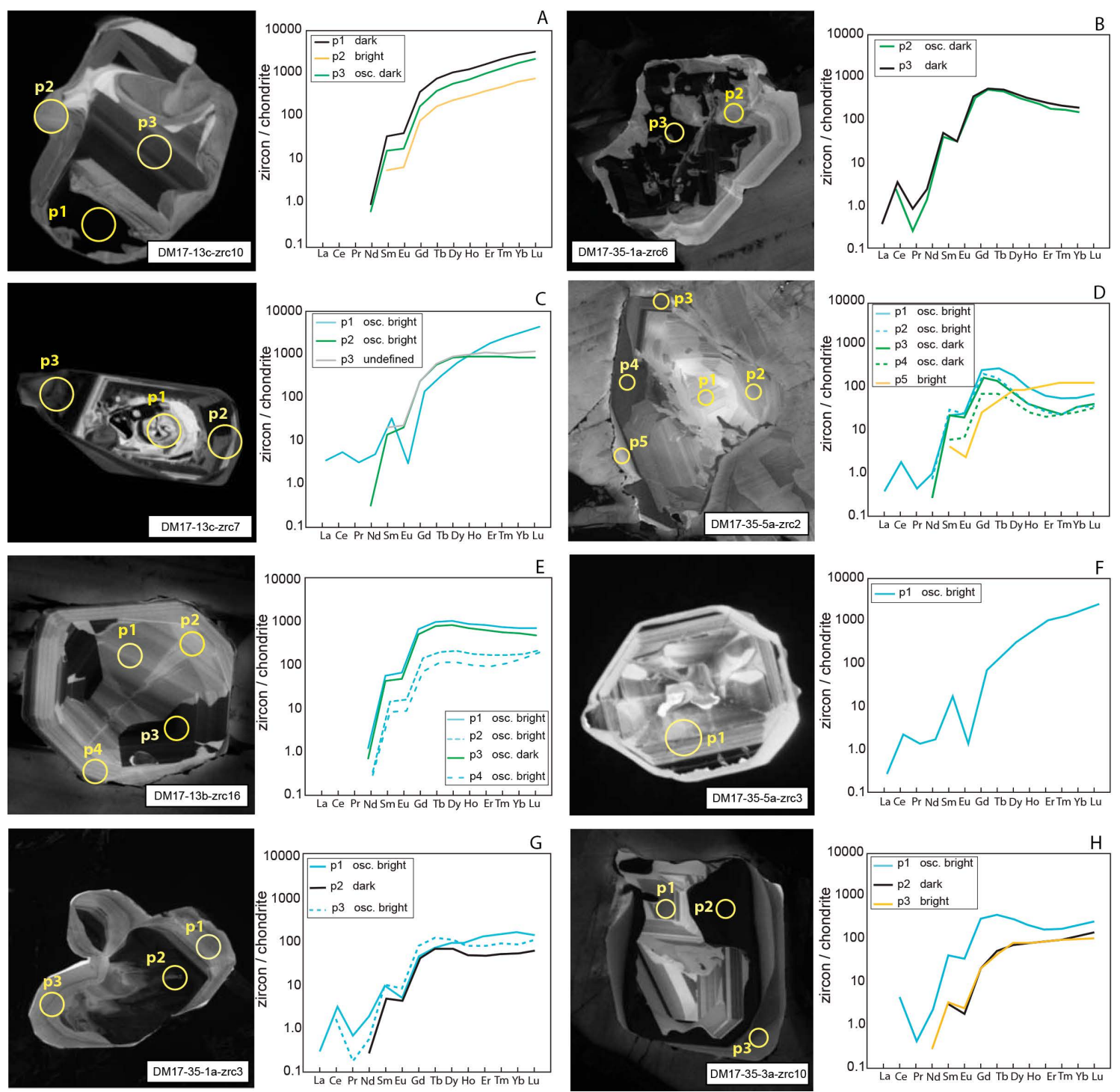

FIGURE 3. REE pattern in selected partially exposed zircon inclusions. Color coding follows that in Figure 2: black $=$ dark, green $=$ oscillatorydark, blue $=$ oscillatory-bright, orange $=$ bright, gray $=$ undefined. Yellow circles are scaled with respect the figure and are $20 \mu \mathrm{m}$ across $($ laser spot).

vs. $D \alpha$ diagram, most of the Alpine domains fall within the broad interpolation line given by Nasdala et al. 2001 (Fig. 6). Nasdala et al. (2001) interpreted the data plotting outside of such interpolation band as the possible effects of thermal annealing in the crystal. In our case, we interpret the few points outside the interpolation band as the result of possible partial annealing of inherited zircon cores (Permian and Caledonian in age) during the Alpine metamorphism.

\section{Effect of metamictization and annealing on Raman shift}

Previous studies have established that partial metamictization can have a major influence on zircon Raman spectra (e.g., Zhang et al. 2000; Geisler et al. 2001; Nasdala et al. 2001; Binvignat et al. 2018). In general, depending on the degree of metamictization, zircon crystals can show a significant Raman peak broadening and frequency shift toward lower wavenumbers with respect to pristine samples taken as reference. Furthermore, Geisler et al.
(2001) pointed out the possible heterogeneous (step-evolution) effect that annealing could have on peak broadening and position.

Based on these considerations, the relationship between the peak width and the Raman shift (Fig. 2) can be exploited as a discriminant between partially metamict/annealed crystals and pristine crystals, even when they are completely buried within their mineral host (see also Zhong et al. 2019). Indeed, differently to phonon wavenumber, variation in the $\Gamma$ of a Raman peak is independent from minor amounts of stress (Binvignat et al. 2018) as those usually recorded in host-inclusion systems. It follows that, when considering zircon inclusions, a buried partially metamict crystal should present $\Gamma$ comparable to partially metamict crystals exposed at the surface and the same is valid for well-crystalline zircon grains. Therefore, we conclude that measurements with $\Gamma$ greater than $5 \mathrm{~cm}^{-1}$ showing an inverse relationship with the Raman shift, represent partially metamict/annealed domains. On the 
TABLE 2. Indicative age and dose of partially exposed zircon inclusions (spots are referred to Fig. 3)

\begin{tabular}{|c|c|c|c|c|c|c|}
\hline$\overline{\text { Sample name }}$ & $\begin{array}{c}\text { CC } \\
\text { domain }\end{array}$ & $\begin{array}{c}\text { Indicative } \\
{ }^{206} \mathrm{~Pb} /{ }^{238} \mathrm{U}\end{array}$ & $\begin{array}{c}\text { Indicative } \\
\text { Age }\end{array}$ & $\begin{array}{c}D_{a} \\
\text { (g/events) }\end{array}$ & $\begin{array}{c}\text { Raman } \\
\text { shift } \\
\left(\mathrm{cm}^{-1}\right)\end{array}$ & $\begin{array}{c}\Gamma \\
\left(\mathrm{cm}^{-1}\right)\end{array}$ \\
\hline DM17-35-3A-zrc8-p1 & osc. dark & 0.04 & Permian & $8.3 \mathrm{E}+17$ & 1007.6 & 5.6 \\
\hline DM17-35-3A-zrc8-p1 & osc. dark & 0.04 & Permian & $8.3 E+17$ & $1007.6(2)$ & $5.6(4)$ \\
\hline DM17-35-3A-zrc8-p2 & osc. bright & 0.08 & Caledonian & $2.2 \mathrm{E}+18$ & $1008.0(3)$ & $3.4(3)$ \\
\hline DM17-35-3A-zrc10-p1 & osc. bright & 0.01 & Alpine & $5.3 \mathrm{E}+16$ & $1007.9(2)$ & $3.9(4)$ \\
\hline DM17-35-3A-zrc10-p2 & dark & 0.01 & Alpine & $2.6 \mathrm{E}+17$ & $1006.7(5)$ & $5.3(3)$ \\
\hline DM17-35-3A-zrc10-p3 & bright & 0.01 & Alpine & $1.2 \mathrm{E}+17$ & $1007.9(4)$ & $3.6(3)$ \\
\hline DM17-35-5A-zrc2-p1 & osc. bright & 0.01 & Alpine & $4.9 \mathrm{E}+16$ & 1009.1(3) & $3.6(4)$ \\
\hline DM17-35-5A-zrc2-p4 & osc. dark & 0.01 & Alpine & $1.6 \mathrm{E}+17$ & $1008.0(3)$ & $4.5(4)$ \\
\hline DM17-35-5A-zrc2-p5 & bright & 0.01 & Alpine & $1.4 \mathrm{E}+17$ & $1008.9(2)$ & $3.5(4)$ \\
\hline DM17-35-1A-zrc6-p2 & osc. dark & 0.01 & Alpine & $3.3 E+17$ & $1008.9(2)$ & $3.5(3)$ \\
\hline DM17-35-1A-zrc6-p3 & dark & 0.01 & Alpine & $3.9 \mathrm{E}+17$ & $1006.6(5)$ & $6.2(3)$ \\
\hline DM17-35-1A-zrc3-p2 & dark & 0.01 & Alpine & $3.7 \mathrm{E}+17$ & $1006.3(3)$ & $5.7(3)$ \\
\hline DM17-35-1A-zrc3-p3 & osc. bright & 0.01 & Alpine & $4.1 \mathrm{E}+17$ & 1007.7(3) & $4.2(2)$ \\
\hline DM17-13-b-zrc13-p1 & osc. bright & 0.01 & Alpine & $1.5 \mathrm{E}+17$ & $1007.9(2)$ & $4.6(4)$ \\
\hline DM17-13-b-zrc13-p2 & osc. bright & 0.01 & Alpine & $1.1 \mathrm{E}+17$ & 1007.7(3) & $4.7(4)$ \\
\hline DM17-13-b-zrc15-p2 & osc bright & 0.01 & Alpine & $8.6 \mathrm{E}+16$ & $1008.3(2)$ & $4.5(4)$ \\
\hline DM17-13-b-zrc15-p3 & osc. bright & 0.01 & Alpine & $4.7 \mathrm{E}+16$ & $1008.3(2)$ & $4.0(3)$ \\
\hline DM17-13-b-zrc16-p1 & osc. bright & 0.01 & Alpine & $1.7 \mathrm{E}+17$ & $1007.9(3)$ & $5.1(3)$ \\
\hline DM17-13-b-zrc16-p2 & osc. bright & 0.01 & Alpine & $8.5 \mathrm{E}+16$ & $1008.2(1)$ & $4.0(4)$ \\
\hline DM17-13-b-zrc16-p3 & osc. dark & 0.01 & Alpine & $2.2 \mathrm{E}+17$ & $1007.3(2)$ & $5.6(4)$ \\
\hline DM17-13-b-zrc16-p4 & osc. bright & 0.01 & Alpine & $5.3 \mathrm{E}+16$ & $1008.3(2)$ & $3.6(5)$ \\
\hline DM17-13-c-zrc7-p1 & osc. bright & 0.04 & Permian & $6.6 \mathrm{E}+17$ & 1009.3(1) & $5.8(3)$ \\
\hline DM17-13-c-zrc7-p2 & osci. dark & 0.01 & Alpine & $1.1 \mathrm{E}+17$ & $1011.3(2)$ & $3.9(3)$ \\
\hline DM17-13-c-zrc7-p3 & undefined & 0.01 & Alpine & $1.3 \mathrm{E}+17$ & $1010.2(3)$ & $4.3(4)$ \\
\hline DM17-13-c-zrc10-p1 & dark & 0.01 & Alpine & $5.2 \mathrm{E}+17$ & $1006.2(5)$ & $10.6(3)$ \\
\hline DM17-13-c-zrc10-p2 & bright & 0.01 & Alpine & $1.4 \mathrm{E}+17$ & $1008.3(2)$ & $3.3(2)$ \\
\hline DM17-13-c-zrc10-p3 & osc. dark & 0.01 & Alpine & $2.3 \mathrm{E}+17$ & $1007.4(3)$ & $5.0(4)$ \\
\hline
\end{tabular}

Note: Uncertainty on the $D_{a}$ is within $14 \%$ based on the $U$ and Th concentration uncertainty.

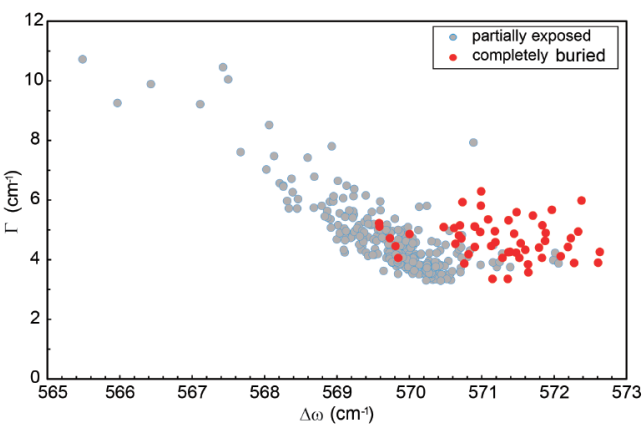

Figure 5. $\Gamma$ of the $B_{1 \mathrm{~g}}$ mode near $1008 \mathrm{~cm}^{-1}$ and $\Delta \omega=\omega_{1008}-\omega_{440}$ of partially exposed vs. selected and completely buried zircon inclusions.

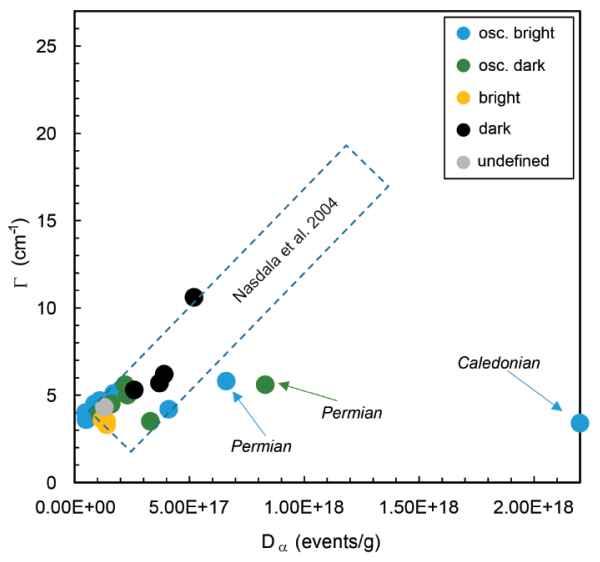

Figure 6. $\Gamma$ of the $B_{1 \mathrm{~g}}$ mode near $1008 \mathrm{~cm}^{-1}$ as function of the effect of increasing metamictization in terms of accumulated radiation dose.

Rubatto and Hermann 2001; Gauthiez-Putallaz et al. 2016).

However, it is difficult to make a rigorous prediction of the effect of metamictization on the determination of the residual pressure of a buried inclusion. For zircon with $\Gamma$ larger than $5 \mathrm{~cm}^{-1}$ there are at least two additional unknown variables:

- The reference wavenumber $\omega_{0}$ of an equally metamict free crystal;

- The phonon mode compressibility $\beta=\left[1 / \omega_{0}\right][d \omega / \mathrm{d} P]$.

Both variables are very sensitive to the accumulated radiation dose $D_{\alpha}$ (e.g., Binvignat et al. 2018). Unfortunately, the dispersion of our data in the established $\Gamma-D_{\alpha}$ trend is too large to give a reliable value of $D_{\alpha}$ and consequently of $\omega_{0}$ and $\beta$ for the purposes of Raman thermobarometry.

\section{Effect of zircon size on Raman shift for partially exposed inclusions}

For completely unstressed pristine crystals, the phonon wavenumber usually is expected not to exceed the values of 1008.5-1009 $\mathrm{cm}^{-1}$ (see Geisler et al. 2001; Binvignat et al. 2018). Nevertheless, our partially exposed grains, even considering only the non-metamict domain in Figure 2, show a larger variation (up to $1011 \mathrm{~cm}^{-1}$ ).

As reported by Campomenosi et al. (2018), partially exposed
FIGURE 4. $\Gamma$ and Raman shift of partially exposed vs. completely buried zircon inclusions. 
inclusions can still preserve a notable stress state in terms of Raman peak shift as a function of the inclusion exposition degree and size. To better clarify this issue, Figure 7 shows a $\Gamma$ vs. $\Delta \omega$ diagram of partially exposed inclusions discriminating between grains with different sizes $(<50$ and $>50 \mu \mathrm{m})$. Note that, in this case, we show only data from the exposed inclusions showing bright and or oscillatory-bright domains at CC because only for such domains can we safely exclude other effects described above. Data from inclusions of different size largely overlap. However, about $20 \%$ of the smaller inclusions have a higher $\Delta \omega$ than the larger one (with one exclusion) and reach a $\Delta \omega$ of $572 \mathrm{~cm}^{-1}$ that are never observed in the larger inclusions. This is in agreement with theoretical predictions (Mazzucchelli et al. 2018) for which, within the same host, small inclusions, when partially exposed, tend to retain more stress, due to the larger surface-to-volume ratio, i.e., larger impact of the host upon relatively larger surface of the inclusion. On the other hand, small inclusions should exhibit less dispersion of the degree of exposure. Hence, the quite large spread of our data is most probably due to the superposition of both effects related to the inclusion size: the surface-to-volume ratio and the degree of exposure.

\section{A new protocol for the selection of zircon inclusions in garnet for elastic thermobarometry}

Based on the above considerations, we propose a simple protocol for selecting zircon inclusions to get reliable residual pressure estimates for elastic thermobarometry.

- Buried crystals must be isolated from other inclusions, section surfaces, host boundary, cracks, and from any other kind of boundaries. This implies that thicker sections (i.e., $300 \mu \mathrm{m}$ ) are better (see also Campomenosi et al. 2018).

- At least 2-3 measurement spots for each crystal moving from core to rim should be performed: this enables detection of eventual structural heterogeneity within the crystal. For this pourpose, it is better to use a spectrometer with a confocal optical microscope to reach the best spatial resolution.

- An instrumental sepctral resultion equal or better than $2 \mathrm{~cm}^{-1}$ should be used and only those inclusions presenting a $\Gamma$ of the $B_{1 \mathrm{~g}}$ mode near $1008 \mathrm{~cm}^{-1}$ smaller than $5 \mathrm{~cm}^{-1}$

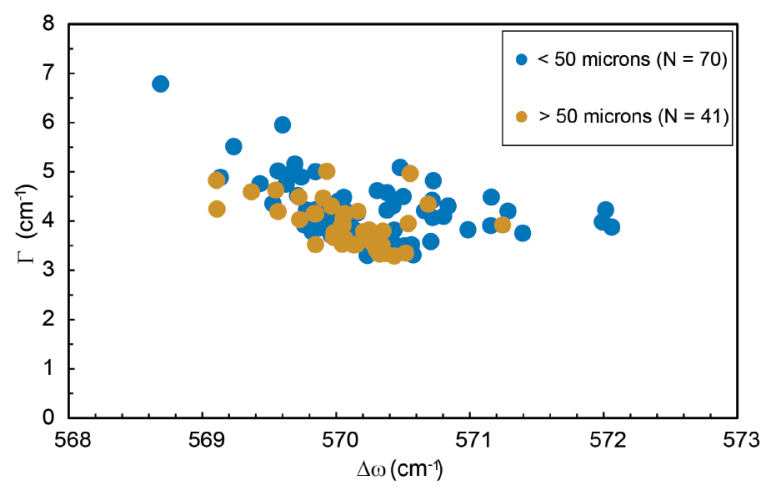

Figure 7. $\Gamma$ of the $B_{1 \mathrm{~g}}$ mode near $1008 \mathrm{~cm}^{-1}$ vs. $\Delta \omega=\omega_{1008}-\omega_{440}$ comparing small $(<50 \mu \mathrm{m})$ and large $(>50 \mu \mathrm{m})$ partially exposed zircon inclusions. The spots refer only to bright and oscillatory bright domains. should be considered. Note that this value represents the summation of the analytical uncertainty (i.e., instrumental spectral resolution) to the minimum value of about $3 \mathrm{~cm}^{-1}$ that has been measured on several grains by the two different spectrometers (see Fig. 4).

- Only inclusions in which the phonon wavenumber and the $\Gamma$ is constant across the entire crystal volume should be selected.

- If $\Gamma$ is constant, but the phonon wavenumber changes across the crystal volume there could be effects due to the shape of the inclusion (Campomenosi et al. 2018). In this case it is reccomended to consider the measurement collected at the center of the crystal to calculate the residual pressure of the inclusion.

- Partially exposed crystals should not be used as reference to calculate residual stress in the buried inclusions: they can be still under a notable residual stress state. It is reccomended using a completely free crystal or large exposed inclusions for which the residual stress state and the metamictization effects are negligible (see for example Fig. 1d).

- Whenever possible, a statistically significant amount of partially exposed inclusions should be selected to double check their textural complexity by CC or CL imaging and their chemistry. As an alternative, imaging and chemical checks should be performed on inclusions that have been exposed after the Raman measurements.

- Chemical and age measurements should be considered as important corollary information to reconstruct the petrogenesis of the zircon inclusions and the garnet host.

\section{IMPLICATIONS}

Zircon inclusions are difficult to manage correctly for elastic thermobarometry applications, and a detailed characterization of the inclusions should be performed before extracting thermobarometric data. In our systematic study, the combination of structural and chemical information obtained by different analytical techniques on partially exposed zircon inclusions allows to reliably predict the structural state of buried crystals for Raman spectroscopic measurements.

Our results provide a solid basis for the selection of reliable zircon inclusions to use for elastic thermobarometry applications. This method, in combination with the already rich tool set that can be applied to zircon [e.g., Ti-in-zircon thermometry (Watson et al. 2006), U-Pb geochronology, oxygen isotopes], will provide an even more detailed characterization of $P-T$ - $t$ fluid and deformation hystory of metamorphic rocks.

\section{ACKNOWLedgments}

Thanks are due to F. Piccoli and to A. Berger (University of Bern) for assistance in the LA-ICP-MS laboratory and the SEM laboratory, respectively, at the Institute of Earth Science of Bern and to N. Waeselmann for assistance in the laboratory of Raman spectroscopy at the University of Hamburg. We are grateful to D. Gatta for manuscript editing and to $\mathrm{M}$. Cisneros and an anonymous reviewer for constructive comments.

\section{FUNDING}

This work was financially supported by the European Research Council (ERC) under the European Union's Horizon 2020 research and innovation program grant agreements 714936 to M. Alvaro. N. Campomenosi acknowledges the University of Genova for funding. D. Rubatto acknowledges the financial support of the Swiss National Science Foundation (project 166280). 


\section{REFERENCES CITED}

Binvignat, F.A.P., Malcherek, T., Angel, R.J., Paulmann, C., Schlüter, J., and Mihailova, B. (2018) Radiation-damaged zircon under high pressures. Physics and Chemistry of Minerals, 45, 981-993.

Campomenosi, N., Mazzucchelli, M.L., Mihailova, B., Scambelluri, M., Angel, R.J., Nestola, F., Reali, A., and Alvaro, M. (2018) How geometry and anisotropy affect residual strain in host-inclusion system: Coupling experimental and numerical approaches. American Mineralogist, 103, 2032-2035.

Chopin, C. (1984) Coesite and pure pyrope in high-grade blueschists of the Western Alps: a first record and some consequences. Contributions to Mineralogy and Petrology, 86, 107-118.

Colombo, M., Chrosch, J., and Salje, E.K. (1999)Annealing metamict zircon: A powder $\mathrm{X}$-ray diffraction study of a highly defective phase. Journal of the American Ceramic Society, 82(10), 2711-2716.

Corfu, F., Hanchar, J.M., Hoskin, P.W.O., and Kinny, P. (2003) Atlas of zircon textures. Reviews in Mineralogy and Geochemistry, 53, 469-500.

Ewing, R.C., Lutze, W., and Weber, W.J. (1995) Zircon: A host-phase for the disposal of weapons plutonium. Journal of Materials Research, 10(2), 243-246.

Ferrando, S., Frezzotti, M.L., Petrelli, M., and Compagnoni, R. (2009) Metasomatism of continental crust during subduction: the UHP whiteschists from the Southern Dora-Maira Massif (Italian Western Alps). Journal of Metamorphic Geology, 27(9), 739-756.

Gauthiez-Putallaz, L., Rubatto, D., and Hermann, J. (2016) Dating prograde fluid pulses during subduction by in situ $\mathrm{U}-\mathrm{Pb}$ and oxygen isotope analysis. Contributions to Mineralogy and Petrology, 171(2), 15.

Gebauer, D., Schertl, H.P., Brix, M., and Schreyer, W. (1997) 35 Ma old ultrahighpressure metamorphism and evidence for very rapid exhumation in the Dora Maira Massif, Western Alps. Lithos, 41(1-3), 5-24.

Geisler, T., and Pidgeon, R.T. (2002) Raman scattering from metamict zircon: comments on "Metamictisation of natural zircon: accumulation versus thermal annealing of radioactivity-induced damage" by Nasdala et al. (2001) (Contributions to Mineralogy and Petrology 141: 125-144). Contributions to Mineralogy and Petrology, 143(6), 750-755.

Geisler, T., Pidgeon, R.T., van Bronswijk, W., and Pleysier, R. (2001) Kinetics of thermal recovery and recrystallization of partially metamict zircon: a Raman spectroscopic study. European Journal of Mineralogy, 13(6), 1163-1176.

Griffin, B.J. (2000) Charge contrast imaging of material growth and defects in environmental scanning electron miscroscopy - linking electron emission and cathodoluminescence. Scanning, 22(4), 234-242.

Hermann, J. (2003) Experimental evidence for diamond-facies metamorphism in the Dora-Maira massif. Lithos, 70(3-4), 163-182.

Hermann, J., Rubatto, D., Korsakov, A., and Shatsky, V.S. (2001) Multiple zircon growth during fast exhumation of diamondiferous, deeply subducted continental crust (Kokchetav massif, Kazakhstan). Contributions to Mineralogy and Petrology, 141, 66-82.

Hoskin, P.W.O., and Rodgers, K.A. (1996) Raman spectral shift in the isomorphous series $\left(\mathrm{Zr}_{1-\mathrm{x}} \mathrm{Hf}_{\mathrm{x}}\right) \mathrm{SiO}_{4}$. European Journal of Solid State and Inorganic Chemistry, 33, 1111-1121.

Hoskin, P.W., and Schaltegger, U. (2003) The composition of zircon and igneous and metamorphic petrogenesis. In J.M. Hanchar and P.W.O. Hoskin, Eds., Zircon, 53, p. 427-467. Review in Mineralogy and Geochemistry, Mineralogical Society of America, Chantilly, Virginia

Katayama, I., and Maruyama, S. (2009) Inclusion study in zircon from ultrahigh-pressure metamorphic rocks in the Kokchetav massif: an excellent tracer of metamorphic history. Journal of the Geological Society, 166, 783-796.

Knittle, E., and Williams, Q. (1993) High-pressure Raman spectroscopy of $\mathrm{ZrSiO}_{4}$ : Observation of the zircon to scheelite transition at $300 \mathrm{~K}$. American Mineralogist, $78,245-252$.
Kroumova, E., Aroyo, M.I., Perez-Mato, J.M., Kirov, A., Capillas, C., Ivantchev, S., and Wondratschek, H. (2010) Bilbao Crystallographic Server: Useful databases and tools for phase-transition studies. Phase Transitions, 76, 155-170.

Kuzmany, H. (2009) Solid-State Spectroscopy. An introduction. Springer-Verlag, Berlin Heidelberg.

Mazzucchelli, M.L., Burnley, P., Angel, R.J., Morganti, S., Domeneghetti, M.C., Nestola, F., and Alvaro, M. (2018) Elastic geothermobarometry: Corrections for the geometry of the host-inclusion system. Geology, 46(3), 231-234.

Nasdala, L., Wenzel, M., Vavra, G., Irmer, G., Wenzel, T., and Kober, B. (2001) Metamictisation of natural zircon: accumulation versus thermal annealing of radioactivityinduced damage. Contributions to Mineralogy and Petrology, 141(2), 125-144.

Nasdala, L., Lengauer, C.L., Hanchar, J.M., Kronz, A., Wirth, R., Blanc, P., and Seydoux-Guillaume, A.M. (2002) Annealing radiation damage and the recovery of cathodoluminescence. Chemical Geology, 191(1-3), 121-140.

Parkinson, C.D., and Katayama, I. (1999) Present-day ultrahigh-pressure conditions of coesite inclusions in zircon and garnet: Evidence from laser Raman microspectroscopy. Geology, 27(11), 979-982.

Paton, C., Hellstrom, J., Paul, B., Woodhead, J., and Hergt, J. (2011) Iolite: freeware for the visualisation and processing of mass spectrometric data. Journal of Analytical Atomic Spectrometry, 26, 2508-2518.

Ríos, S., Salje, E.K., Zhang, M., and Ewing, R.C. (2000) Amorphization in zircon: evidence for direct impact damage. Journal of Physics: Condensed Matter, 12(11), 2401

Rubatto, D., and Gebauer, D. (2000) Use of cathodoluminescence for U-Pb zircon dating by ion microprobe: some examples from the Western Alps. In M. Pagel, V. Barbin, P. Blanc, and D. Ohnenstetter, Eds., Cathodoluminescence in Geosciences, p. 373-400. Springer.

Rubatto, D., and Hermann, J. (2001) Exhumation as fast as subduction?. Geology, 29(1), 3-6.

Rubatto, D., and Hermann, J. (2007) Zircon behaviour in deeply subducted rocks Elements, 3, 31-35.

Stangarone, C., Angel, R.J., Prencipe, M., Campomenosi, N., Mihailova, B., and Alvaro, M. (2019) Measurement of strains in zircon inclusions by Raman spectroscopy. European Journal of Mineralogy, 31 (4), 685-694.

Watt, G.R., Kinny, P.D., and Griffin, B.J. (2000) Charge contrast imaging of geological materials in the environmental scanning electron microscope. American Mineralogist, 85, 1784-1794.

Watson, E.B., Wark, D.A., and Thomas, J.B. (2006) Crystallization thermometers for zircon and rutile. Contributions to Mineralogy and Petrology, 151, 413-433.

Woodhead, J.D., Hellstrom, J., Hergt, J.M., Greig, A., and Maas, R. (2007) Isotopic and elemental imaging of geological materials by laser ablation inductively coupled plasma-mass spectrometry. Geostandards and Geoanalytical Research, 31, 331-343.

Ye, K., Yao, Y.P., Katayama, I., Cong, B.L., Wang, Q.C., and Maruyama, S. (2000b) Large areal extent of ultrahigh-pressure metamorphism in the Sulu ultrahigh-pressure terrane of East China: New implications from coesite and omphacite inclusions in zircon of granitic gneiss. Lithos, 52, 157-164.

Zhang, M., Salje, E.K., Capitani, G.C., Leroux, H., Clark, A.M., Schlüter, J., and Ewing, R.C. (2000) Annealing of-decay damage in zircon: a Raman spectroscopic study. Journal of Physics: Condensed Matter, 12(13), 3131.

Zhong, X., Andersen, N.H., Dabrowski, M., and Jamtveit, B. (2019) Zircon and quartz inclusions in garnet used for complementary Raman thermobarometry: application to the Holsnøy eclogite, Bergen Arcs, Western Norway. Contributions to Mineralogy and Petrology, 174(6), 50.

ManusCript ReCEIVEd August 6, 2019

MANUSCRIPT ACCEPTED NOVEMBER 20, 2019

ManusCRIPT handled by G. Diego Gatta 